\title{
ALGEBRAIC CURVES, RIEMANN SURFACES AND KLEIN SURFACES WITH NO NON-TRIVIAL AUTOMORPHISMS OR SYMMETRIES
}

\author{
PETER TURBEK \\ Department of Mathematics, Statistics, and Computer Science, \\ Purdue University Calumet, Hammond, IN 46323, USA \\ (turbek@nwi.calumet.purdue.edu)
}

(Received 14 December 1999)

Dedicated to the memory of Sheela Phansalkar (1966-1990)

\begin{abstract}
The explicit defining equations of a new family of curves whose members have a trivial automorphism group are given. Each member is defined for characteristic zero and all but a finite number of characteristics greater than zero. This family, in conjunction with a previously appearing family of the author's, provides explicit examples of algebraic curves which possess only the trivial automorphism for each genus greater than three. The family is then used to construct Riemann surfaces without anticonformal automorphisms and Klein surfaces with no non-trivial automorphisms.
\end{abstract}

Keywords: algebraic curves; automorphisms; Riemann surface; Klein surface

AMS 2000 Mathematics subject classification: Primary 14H37; 30F50; 30F99

\section{Introduction}

It is well known that a generic curve or Riemann surface of genus greater than two has a trivial automorphism group, but there are few explicit examples with their defining equations in the literature. Cronheim [3] produced examples of curves with a trivial automorphism group which are defined for characteristic $p>0$, however the definition of each curve depends on the chosen $p$. Thus each example is shown to yield a trivial automorphism group for only a single characteristic. In [1] , Accola determined a procedure which yields examples of Riemann surfaces with trivial automorphism groups. Madden and Valentini [7], in the course of showing that every group can be realized as the full automorphism group of an algebraic function field of positive characteristic, explicitly constructed a family of curves with trivial automorphism groups, defined for arbitrary characteristic (including 0). Each of these curves has genus 13. Mednykh [8] and Everitt [4] produced families of Riemann surfaces without automorphisms by using a fundamental region for each surface or its associated Fuchsian group, however they did not find defining algebraic equations for the families. 
In [10], the author presented an explicit family of curves whose members possess trivial automorphism groups. The doubly infinite family presented there contains curves with a trivial automorphism group for each genus $g=(n-1)(m-1) / 2$, where $m$ and $n$ are relatively prime integers which satisfy $n>m+1>3$. In addition, each member of the family excludes only finitely many characteristics; the only assumption made is that the characteristic equals zero, or does not divide $(m-1) m n$. It is easy to see that this family yields examples of curves with a trivial automorphism group for each genus greater than three of the form $3 k$ or $3 k+1$ (by choosing $m=3$ ), but not every genus of the form $3 k+2$ is represented (consider $g=5$ ). In this paper, for each genus of the form $g=n(m-1) / 2$, where $n-1$ and $m$ are relatively prime integers satisfying $n>m+1>3$, we present an $m$ parameter family of curves with trivial automorphism groups. The only restriction is that the characteristic of the field does not divide $n(n-1) m$. Clearly this family contains examples for each genus greater than three of the form $3 k$ or $3 k+2$, so this family in conjunction with the family presented in $[\mathbf{1 0}]$ furnish examples of curves with trivial automorphism groups for each genus greater than three.

After proving that members of the above-mentioned family have a trivial automorphism group, we determine conditions which will ensure that, when defined over $\mathbb{C}$, its members have no anticonformal automorphisms. We then show how the family can be used to give explicit equations of Klein surfaces which possess no non-trivial automorphisms.

In the following section we construct the above-mentioned family of curves. Each curve $C$ in the family has a Weierstrass point $P$ with a unique gap sequence; thus, an automorphism of $C$ must fix $P$. It is proved that only the identity automorphism fixes $P$, thus the automorphism group of $C$ is trivial.

\section{Construction}

We adhere to the notation used in [10]. In particular the order of a function $f$ at a point $R$ of a curve is denoted by $\operatorname{ord}_{R} f$, with a similar notation used for differentials. If $D$ is a divisor, the set of functions $f$ such that $(f)+D \geqslant 0$ is denoted by $L(D)$. We say that $m$ is a gap at $R$ if there is no function $f$ with $(f)_{\infty}=m R$. If $m$ is a gap at $R$, it is a gap at the image of $R$ under an automorphism of the curve. If $\sigma$ is an automorphism of a curve, we denote the induced automorphism of the function field of the curve by $\sigma^{*}$.

Let $k$ denote an arbitrary algebraically closed field and let $\mathbb{A}^{2}$ denote two-dimensional affine space with coordinates $(x, y)$. Let $n>m+1$ be positive integers such that $n-1$ and $m$ are relatively prime and $m \geqslant 3$. We assume that the characteristic of the field does not divide $n(n-1) m$. Let $C$ denote the affine locus of the equation

$$
f(x, y)=y^{n}+y p(x)+a_{0} x=0 .
$$

In (2.1), $p(x)$ is any element of $k[x]$ of degree $m$ such that $x$, but not $x^{2}$, divides $p(x)$, and the coefficient of $x^{m-1}$ is 0 . In addition, $a_{0} \in k^{*}$ is chosen to make (2.1) non-singular in $\mathbb{A}^{2}$. 
The requirement that $C$ be non-singular in $\mathbb{A}^{2}$ is easily accomplished. Recall that singularities occur at points where $f=0, f_{x}=0$ and $f_{y}=0$. From (2.1) we have

$$
f_{x}=y p^{\prime}(x)+a_{0}, \quad f_{y}=n y^{n-1}+p(x) .
$$

At singular points $n f-y f_{y}=0$. This yields

$$
y=\frac{-n a_{0} x}{(n-1) p(x)} .
$$

Substituting this into $f_{x}=0$, we obtain

$$
(n-1) p(x)-n x p^{\prime}(x)=0 .
$$

Let $x_{1}, x_{2}, \ldots, x_{m}$ be the not necessarily distinct solutions of (2.4). Note that these values are independent of $a_{0}$. Thus, singular points have coordinates

$$
\left(x_{i}, \frac{-n a_{0} x_{i}}{(n-1) p\left(x_{i}\right)}\right) \text {. }
$$

Now choose $a_{0}$ such that none of these points satisfy $f_{y}=0$. Then $C$ is non-singular.

Observe that $x$ divides $p(x)$ and $a_{0} x$. Thus, applying Eisenstein's criterion, we obtain that (2.1) is the irreducible polynomial for $y$ over $k(x)$.

We note from (2.1) that $x$ has a zero of order $n$ at $(0,0)$, and $y$ has a zero of order 1 at $(0,0)$ as its unique zero on $C$.

Let $\mathbb{P}^{2}$ denote two-dimensional projective space over $k$ with homogeneous coordinates $(X, Y, Z)$. Let $\bar{C}$ denote the locus in $\mathbb{P}^{2}$ of the homogenization of (2.1), namely

$$
F(X, Y, Z)=Y^{n}+Y P(X, Z)+a_{0} X Z^{n-1} .
$$

Here $P(X, Z)$ is a homogeneous polynomial of degree $n-1$ such that $P(x, 1)=p(x)$. Setting $Z=0$ in $(2.6)$, we obtain that $F(X, Y, 0)=Y^{n}$, so $(1,0,0)$ is the only point at infinity. It is easily seen to be a singular point, and we now examine this point closely.

Let $C^{\prime}$ be the non-singular projective model for (2.6). Note that $x=X / Z$ and $y=Y / Z$ have pole divisors of degrees $n$ and $m$, respectively, on $C^{\prime}$. We note that at $(1,0,0)$ the function $1 / x=Z / X$ has a zero. Thus $x$ has a pole there, and thus it has a pole at any point on $C^{\prime}$ which lies over $(1,0,0)$. Since $y$ has no pole in $\mathbb{A}^{2}$, it must have a pole at some point $P \in C^{\prime}$ which lies over $(1,0,0)$. Comparing the orders of the terms in (2.1) at the point $P$, we obtain $n\left(\operatorname{ord}_{P} y\right)=\operatorname{ord}_{p} y+m\left(\operatorname{ord}_{P} x\right)$. Thus $(n-1) \operatorname{ord}_{P} y=m\left(\operatorname{ord}_{P} x\right)$. Since $n-1$ and $m$ are relatively prime, $m$ divides $\operatorname{ord}_{P} y$, and thus $-m=\operatorname{ord}_{P} y$ and $1-n=\operatorname{ord}_{P} x$. Since $n$ is the degree of the pole divisor of $x$, we see that there is exactly one other point $Q \neq P$ on $C^{\prime}$ which lies over $(1,0,0)$. Since $(0,0)$ is the unique point on $C$ where $y$ has a zero, and $y$ has order one there, we obtain $\operatorname{ord}_{Q} y=m-1$. Thus $P$ and $Q$ are the unique points of $C^{\prime}$ which lie over $(1,0,0)$ and we may view $C^{\prime}$ as $C \bigcup\{P, Q\}$. In particular we have $(x)=n(0,0)+(1-n) P-Q$, and $(y)=(0,0)-m P+(m-1) Q$.

Lemma 2.1. Let $\omega=\mathrm{d} x / f_{y}=-\mathrm{d} y / f_{x}$. Then $(\omega)=(m n-n-m) P+(m-2) Q$ and the genus of $C^{\prime}$ is $g=n(m-1) / 2$. 
Proof. If $x-a$ is a local parameter at $(a, b)=R \in C$, then $f_{y}(R) \neq 0$. Similarly, if $y-b$ is a local parameter, then $f_{x}(R) \neq 0$. Thus $\omega$ has order 0 at all points of $C$. At $P$ we have $\operatorname{ord}_{P}(\omega)=\operatorname{ord}_{P}\left(-\mathrm{d} y / f_{x}\right)=m n-n-m$. At $Q, \operatorname{ord}_{Q}(\omega)=\operatorname{ord}_{Q}\left(\mathrm{~d} x / f_{y}\right)=m-2$. This proves the first part of the lemma. Recall that the degree of a differential is $2 g-2$. Thus $g=n(m-1) / 2$.

\subsection{Gap sequences at points of $C^{\prime}$}

Let $D=2 g+1=n(m-1)+1$. We determine the gap sequence at $P$ and a basis for $L(D P)$. We require the following proposition.

Proposition 2.2. Let $N$ and $M$ denote positive integers and let $(N, M)=1$. Then

$$
\sum_{i=1}^{M-1}\left[\frac{i N}{M}\right]=\frac{(N-1)(M-1)}{2} .
$$

Proof. See $[\mathbf{9}]$.

Lemma 2.3. A basis for $L(D P)$ is

$$
T=\left\{x^{i} y^{j} \mid i(n-1)+j m \leqslant D, i \geqslant 0, j \geqslant 0 \text { and if } i>0, \text { then } j>0\right\} .
$$

Proof. Note that the element $x^{i} y^{j} \in T$ has a pole of order $i(n-1)+j m$ at $P$, and no pole at $Q$, so $T \subset L(D P)$. We calculate which positive integers $t \leqslant D$ are not orders of poles of elements of $T$. Since $n-1$ and $m$ are relatively prime, $0, n-1,2(n-1), \ldots,(m-$ $1)(n-1)$ form a complete set of residues $\bmod m$. Thus, given $t \leqslant D$ there is an $i$ with $0 \leqslant i \leqslant m-1$ such that $t \equiv i(n-1) \bmod m$. If $t \geqslant i(n-1)+m$, there is a positive $j$ such that $t=i(n-1)+j m$, so $t$ is the order of a pole of an element of $T$. On the other hand, if $t<i(n-1)+m$, no element of $T$ will have a pole of order $t$, since only elements of the form $x^{i} y^{j}$ will have poles of orders congruent to $t$. Thus the number of positive integers $t \leqslant D$ with $t \equiv i(n-1) \bmod m$ which are not orders of poles of elements of $T$ equals the number of integers congruent to $i(n-1)$ and less than $i(n-1)+m$. This value is easily seen to be $[i(n-1) / m]+1$ if $i>0$ and 0 if $i=0$. From Proposition 2.2,

$$
\sum_{i=1}^{m-1}\left[\frac{i(n-1)}{m}\right]=\frac{(n-2)(m-1)}{2} .
$$

Thus there are exactly $(n-2)(m-1) / 2+m-1=n(m-1) / 2=g$ integers $t \leqslant D$ which are not orders of elements of $T$. Since a point has precisely $g$ gaps, each of which is less than $2 g<2 g+1=D$, and we have determined that the set $T$ yields precisely $g$ gaps, we have that $T$ spans $L(D P)$.

Recall the definition of $\omega$ from Lemma 2.1 , and let $W=(\omega)=(n m-n-m) P+(m-$ 2) $Q$. To compute gaps at other points of $C^{\prime}$ we use the following lemma.

Lemma 2.4. Let $t$ be a positive integer. Then $t$ is a gap at $R \in C^{\prime}$ if and only if $L(W-(t-1) R) \neq L(W-t R)$. 
Proof. A trivial consequence of the Riemann-Roch Theorem $[\mathbf{5}, \mathbf{6}]$ applied to $L((t-1) R)$ and $L(t R)$.

Lemma 2.5. Let $R=(a, b) \in C$. Then $\operatorname{ord}_{R}(y-b)<m$.

Proof. We may assume that $R \neq(0,0)$, since the divisor $(y)$ implies the truth of the lemma at $(0,0)$. Since the pole divisor of $y$ has degree $m$, it is sufficient to show that $\operatorname{ord}_{R}(y-b) \neq m$. If this were not the case, then $(a, b)$ would be the unique point of $C$ where $y$ has the value $b$. Thus, substituting $b$ for $y$ in $(2.1)$, we obtain $0=b^{n}+b p(x)+a_{0} x=$ $c(x-a)^{m}$ for some $c \in k^{*}$. This contradicts that the coefficient of $x^{m-1}$ in $p(x)$ is zero.

Lemma 2.6. Let $R=(a, b) \in C$. Then $m$ is a gap at $R$.

Proof. From Lemma 2.4 it is sufficient to show that $L(W-(m-1)) R \neq L(W-m R)$, in other words, that there is a function $g \in L(W)$ with $\operatorname{ord}_{R}(g)=m-1$. If $y-b$ is a local parameter at $R$, let $g=(y-b)^{m-1}$. Then $\operatorname{ord}_{R}(g)=m-1$ and $-\operatorname{ord}_{P}(g)=(m-1) m<$ $(m-1)(n-1)=m n-n-m+1$, so $g \in L(W)$. If $\operatorname{ord}_{R}(y-b)=r>1$, then $(x-a)$ is a local parameter at $R$. In addition, Lemma 2.5 yields that $r<m$. In this case define $g=(y-b)(x-a)^{m-1-r}$. Then $\operatorname{ord}_{R}(g)=m-1$ and $g \in L(W)$, since ord $(g)=r$ and $-\operatorname{ord}_{P}(g)=m+(m-1-r)(n-1) \leqslant m n-m-n$. Thus $m$ is a gap at all points of $C$.

Lemma 2.7. $m$ is a gap at $Q$.

Proof. Again applying Lemma 2.4 it is sufficient to show that $L(W-(m-1) Q) \neq$ $L(W-m Q)$, in other words, that there exists a $g \in L((n m-n-m) P)$ such that $\operatorname{ord}_{Q} g=1$. In this case $g=y x^{m-2}$ has the required properties. Thus $m$ is a gap at $Q$.

Theorem 2.8. $P$ is the unique point of $C^{\prime}$ for which $m$ is not a gap.

Proof. This follows directly from Lemmas 2.3, 2.6 and 2.7.

\subsection{The automorphism group of $C^{\prime}$}

Theorem 2.8 implies that any automorphism of $C^{\prime}$ must fix $P$. We now prove that the only automorphism of $C^{\prime}$ which fixes $P$ is the identity automorphism, thus the automorphism group of $C^{\prime}$ is trivial. In order to simplify the calculations we first make a birational change of coordinates. Recalling the form of $p(x)$ we rewrite $(2.1)$ as

$$
y^{n}+y\left(a_{m} x^{m}+a_{m-2} x^{m-2}+\cdots+a_{1} x\right)+a_{0} x=0 .
$$

Thus

$$
y^{n+m-1}+a_{m}(x y)^{m}+a_{m-2} y^{2}(x y)^{m-2}+\cdots+a_{1} y^{m-1}(x y)+a_{0} y^{m-2}(x y)=0 .
$$

Letting $t=x y$ we obtain

$$
y^{n+m-1}+a_{m} t^{m}+a_{m-2} y^{2} t^{m-2}+\cdots+a_{1} y^{m-1} t+a_{0} y^{m-2} t=0 .
$$


Thus

$$
t^{m}+b_{m-2} y^{2} t^{m-2}+\cdots+b_{2} y^{m-2} t^{2}+\left(b_{1} y^{m-1}+b_{0} y^{m-2}\right) t+b^{\prime} y^{n+m-1}=0,
$$

where $b_{i}=a_{i} / a_{m}$ and $b^{\prime}=1 / a_{m}$. Note that $(t)=(n+1)(0,0)+(1-n-m) P+(m-2) Q$. Since the pole divisor of $y$ has degree $m$, we have $\left[k\left(C^{\prime}\right): k(y)\right]=m$, thus $(2.13)$ is the irreducible polynomial for $t$ over $k(y)$. Note that $y$ and $t$ have poles precisely at $P$, and their respective orders are $m$ and $n+m-1$. Considering the basis $T$ in (2.8) we conclude that if $\sigma \in \operatorname{Aut}\left(C^{\prime}\right)$, then $\sigma^{*}(y)=\alpha y+\beta$, and $\sigma^{*}(t)=h(y)+\gamma t$, where $\alpha, \gamma \in k^{*}, \beta \in k$, and $h(y) \in k[y]$ has degree less than $(m+n-1) / m$. Thus

$$
\begin{aligned}
0=(h(y)+ & \gamma t)^{m}+b_{m-2}(\alpha y+\beta)^{2}(h(y)+\gamma t)^{m-2}+\cdots+b_{2}(\alpha y+\beta)^{m-2}(h(y)+\gamma t)^{2} \\
& +\left(b_{1}(\alpha y+\beta)^{m-1}+b_{0}(\alpha y+\beta)^{m-2}\right)(h(y)+\gamma t)+b^{\prime}(\alpha y+\beta)^{n+m-1} .
\end{aligned}
$$

In particular, (2.14) is an irreducible polynomial for $t$ over $k(y)$. By our assumptions on the characteristic of $k,(2.14)$ has a non-zero coefficient for $t^{m-1}$ if $h(y)$ is not the zero polynomial, contradicting (2.13). Thus $h(y)=0$, so

$$
\begin{aligned}
0=(\gamma t)^{m}+b_{m-2} & (\alpha y+\beta)^{2}(\gamma t)^{m-2}+\cdots+b_{2}(\alpha y+\beta)^{m-2}(\gamma t)^{2} \\
& +\left(b_{1}(\alpha y+\beta)^{m-1}+b_{0}(\alpha y+\beta)^{m-2}\right)(\gamma t)+b^{\prime}(\alpha y+\beta)^{n+m-1} .
\end{aligned}
$$

Since (2.15) has $b^{\prime} \beta^{n+m-1}$ as its constant term, we see from (2.13) that $\beta=0$. Thus

$$
\begin{aligned}
0=(\gamma t)^{m}+b_{m-2}(\alpha y)^{2}(\gamma t)^{m-2} & +\cdots+b_{2}(\alpha y)^{m-2}(\gamma t)^{2} \\
& +\left(b_{1}(\alpha y)^{m-1}+b_{0}(\alpha y)^{m-2}\right)(\gamma t)+b^{\prime}(\alpha y)^{n+m-1} .
\end{aligned}
$$

Comparing (2.13) and (2.16) we conclude

$$
\gamma^{m} b_{1} y^{m-1} t=b_{1}(\alpha y)^{m-1} \gamma t, \quad \gamma^{m} b_{0} y^{m-2} t=b_{0}(\alpha y)^{m-2} \gamma t .
$$

This easily forces $\alpha=1$, which forces $\gamma=1$. Thus the automorphism group of $C^{\prime}$ is trivial.

\section{Symmetries and Klein surfaces}

We now assume that $C^{\prime}$ is defined over the complex numbers $\mathbb{C}$ and determine conditions on the defining equation for $C^{\prime}$ which ensure that it possesses no anticonformal automorphisms. Recall that there is a one-to-one correspondence between the anticonformal automorphisms of the Riemann surface $C^{\prime}$ and the automorphisms of $\mathbb{C}(x, y)=\mathbb{C}(t, y)$ which fix $\mathbb{R}$ but not $\mathbb{C}$. Recall that anticonformal automorphisms preserve the orders of functions at the points of the surface.

Consider (2.13) and let $\sigma$ now denote an anticonformal automorphism. For exactly the same reasons as in the conformal case, $\sigma^{*}(y)=\alpha y+\beta$, and $\sigma^{*}(t)=h(y)+\gamma t$, where $\alpha, \gamma \in \mathbb{C}^{*}, \beta \in \mathbb{C}$, and $h(y) \in \mathbb{C}[y]$ has degree less than $(m+n-1) / m$. If $\sigma$ is an 
anticonformal automorphism, then (2.13) yields that

$$
\begin{array}{r}
0=(h(y)+\gamma t)^{m}+\bar{b}_{m-2}(\alpha y+\beta)^{2}(h(y)+\gamma t)^{m-2}+\cdots+\bar{b}_{2}(\alpha y+\beta)^{m-2}(h(y)+\gamma t)^{2} \\
+\left(\bar{b}_{1}(\alpha y+\beta)^{m-1}+\bar{b}_{0}(\alpha y+\beta)^{m-2}\right)(h(y)+\gamma t)+\bar{b}^{\prime}(\alpha y+\beta)^{n+m-1} .
\end{array}
$$

In particular, (3.1) is an irreducible polynomial for $t$ over $\mathbb{C}(y)$. Note that (3.1) has a non-zero coefficient of $t^{m-1}$ if $h(y)$ is not the zero polynomial, contradicting (2.13). Thus $h(y)=0$, so

$$
\begin{aligned}
0=(\gamma t)^{m}+\bar{b}_{m-2} & (\alpha y+\beta)^{2}(\gamma t)^{m-2}+\cdots+\bar{b}_{2}(\alpha y+\beta)^{m-2}(\gamma t)^{2} \\
& +\left(\bar{b}_{1}(\alpha y+\beta)^{m-1}+\bar{b}_{0}(\alpha y+\beta)^{m-2}\right)(\gamma t)+\bar{b}^{\prime}(\alpha y+\beta)^{n+m-1} .
\end{aligned}
$$

Since (3.2) has $\bar{b}^{\prime} \beta^{n+m-1}$ as its constant term, we see from (2.13) that $\beta=0$. Thus

$$
\begin{aligned}
0=(\gamma t)^{m}+\bar{b}_{m-2}(\alpha y)^{2}(\gamma t)^{m-2} & +\cdots+\bar{b}_{2}(\alpha y)^{m-2}(\gamma t)^{2} \\
& +\left(\bar{b}_{1}(\alpha y)^{m-1}+\bar{b}_{0}(\alpha y)^{m-2}\right)(\gamma t)+\bar{b}^{\prime}(\alpha y)^{n+m-1} .
\end{aligned}
$$

Comparing (2.13) and (3.3) we conclude that $\gamma^{m} b^{\prime}=\bar{b}^{\prime} \alpha^{n+m-1}$ and

$$
\gamma^{m} b_{1} y^{m-1} t=\bar{b}_{1}(\alpha y)^{m-1} \gamma t, \quad \gamma^{m} b_{0} y^{m-2} t=\bar{b}_{0}(\alpha y)^{m-2} \gamma t .
$$

This yields that

$$
\alpha=\frac{\bar{b}_{0} b_{1}}{b_{0} \bar{b}_{1}}
$$

and

$$
\gamma=\frac{b_{1} \bar{b}^{\prime} \alpha^{n}}{\bar{b}_{1} b^{\prime}}
$$

For $i$ satisfying $2 \leqslant i \leqslant m-2,(2.13)$ and (3.3) also yield that $(\gamma / \alpha)^{i}=\bar{b}_{m-i} / b_{m-i}$. To ensure that $C^{\prime}$ possesses no anticonformal automorphisms, it is sufficient to choose a value for one $b_{m-i}$ for which this is not true. Clearly such values can always be chosen. Therefore, with such a choice of $b_{m-i}, C^{\prime}$ possesses no anticonformal automorphisms. Note that in terms of the original equation (2.10), to ensure that $C^{\prime}$ has no anticonformal automorphisms, it is sufficient to define one $a_{m-i}$ for an $i$ with $2 \leqslant i \leqslant m-2$ so that

$$
\left(\frac{a_{0}}{\bar{a}_{0}}\left(\frac{\bar{a}_{0} a_{1}}{a_{0} \bar{a}_{1}}\right)^{n}\right)^{i} \neq \frac{\bar{a}_{m-i} a_{m}}{a_{m-i} \bar{a}_{m}} .
$$

We now show how the family constructed in $\S 2$ can be modified to yield the defining equations of Klein surfaces with a trivial automorphism group. It is well known that the categories of algebraic function fields over $\mathbb{C}$ and the category of Riemann surfaces are functorially equivalent. In a similar manner, the categories of algebraic function fields over $\mathbb{R}$ and the category of compact Klein surfaces are functorially equivalent $[\mathbf{2}]$. In 
particular, if a Klein surface $S$, corresponding to the algebraic function field $E$, possesses a non-trivial automorphism, then $E$ possesses a non-trivial automorphism which fixes $\mathbb{R}$ (but not necessarily $\mathbb{C}$ ). In addition, $\mathrm{i}=\sqrt{-1} \in E$, if and only if $S$ is a Riemann surface.

We now construct a family of Klein surfaces with trivial automorphism groups. Let $f(x, y)$ be defined as in $(2.1)$ over $\mathbb{C}$, with the appropriate restrictions on $p(x)$ and $a_{0}$ so that the resulting Riemann surface possesses no non-trivial automorphisms. Assume, in addition, that the coefficients of $f(x, y)$ are chosen to be real numbers. Recall that it was shown that $f(x, y)$ is the irreducible polynomial for $y$ over $\mathbb{C}(x)$ and thus $[\mathbb{C}(x, y)$ : $\mathbb{C}(x)]=n$. This implies that $[\mathbb{R}(x, y): \mathbb{R}(x)]=n$, that $i \notin \mathbb{R}(x, y)$, and that $\mathbb{C}(x, y)=$ $\mathbb{R}(x, y)(i)$. Let $S$ be the compact Klein surface corresponding to $E:=\mathbb{R}(x, y)$. We now show that $E$ possesses no field automorphism which fixes $\mathbb{R}$. Assume $\phi: E \rightarrow E$ is a field automorphism which fixes $\mathbb{R}$. Then $\phi$ extends to an automorphism of the polynomial ring $E[z]$ by defining $\phi(z)=z$. Since $\mathbb{C}(x, y) \cong E[z] /\left(z^{2}+1\right), \phi$ extends to a non-trivial automorphism of $\mathbb{C}(x, y)$, which is the identity on $\mathbb{C}$. This contradicts that $\mathbb{C}(x, y)$ was proved in $\S 2$ to possess no non-trivial automorphisms.

Acknowledgements. I thank Emilio Bujalance for his helpful comments concerning this paper.

\section{References}

1. R. D. M. Accola, Strongly branched coverings of closed Riemann surfaces, Proc. Am. Math. Soc. 26 (1970), 315-322.

2. E. Bujalance, J. J. Etayo, J. M. Gamboa and G. Gromadzki, Automorphism groups of compact bordered Klein surfaces. A combinatorial approach, Lecture Notes in Mathematics, vol. 1439 (Springer, 1990).

3. A. Cronheim, Ein Funktionenkörper von Primzahlcharakteristik ohne Automorphismen, Math. Nachr. 18 (1958), 99-105.

4. B. Everitt, A family of conformally asymmetric Riemann, Glasgow Math. J. 39 (1997), 221-225.

5. H. M. Farkas and I. Kra, Riemann surfaces, 2nd edn, Graduate Texts in Mathematics, no. 71 (Springer, 1991).

6. W. Fulton, Algebraic curves, an introduction to algebraic geometry (W. A. Benjamin, New York, 1969).

7. D. J. Madden And R. C. VAlentini, The group of automorphisms of algebraic function fields, J. Reine Angew. Math. 343 (1983), 162-168.

8. A. MEDNYKH, Hyperbolic Riemann surfaces with the trivial group of automorphisms, in Deformations of mathematical structures (ed. J. Lawrynowicz), pp. 115-125, (Kluwer, 1988).

9. I. Niven And H. Zuckerman, An introduction to the theory of numbers, 2nd edn (Wiley, 1966).

10. P. Turbek, An explicit family of curves with trivial automorphim groups, Proc. Am. Math. Soc. 122 (1994), 657-664. 